\title{
METODOLOGIA DE PESQUISA PARA ENGENHARIAS: UMA ANÁLISE
}

Ricardo Júnior de Oliveira Silva - ricardojrosilva@gmail.com

UNINTER, Escola Superior Politécnica

Rua Luiz Xavier, 103

80021-980 - Curitiba - Paraná

Dayse Mendes-dayse.m@uninter.com

UNINTER, Escola Superior Politécnica

Rua Luiz Xavier, 103

80021-980 - Curitiba - Paraná

Jéssika Alvares Coppi Arruda Gayer-jessika.c@uninter.com

UNINTER, Escola Superior Politécnica

Rua Luiz Xavier, 103

80021-980 - Curitiba - Paraná

Diego do Nascimento Otto - diegootto45@gmail.com

UNINTER, Escola Superior Politécnica

Rua Luiz Xavier, 103

80021-980 - Curitiba - Paraná

Resumo: O presente artigo, em sua fase atual, faz uma análise dos métodos científicos mais utilizados por pesquisadores engenheiros em suas pesquisas. Para tanto, levantou-se artigos na literatura, selecionou-se os métodos de maior utilização e analisou-se o porquê de serem escolhidos pela maioria dos engenheiros ao realizarem pesquisa. A pergunta que moveu este projeto foi a seguinte: "Quais os métodos de pesquisa científicos são mais utilizados por pesquisadores engenheiros?" A partir disso, observou-se que a maior parte dos estudos encontrados são feitos a partir de questionários elou entrevistas, método estatístico quantitativo, e por alguns, revisão sistemática da literatura. Observou-se também que há certa dificuldade por parte dos engenheiros ao fazerem pesquisa bem como em entender a diferença entre Metodologia de pesquisa científica e Resultados e discussões. Com isso, concluiu-se que há necessidade de estabelecer mais claramente a distinção entre metodologia científica de pesquisa e método de resolução de problemas já ao longo do curso de graduação, e estabelecer uma caracterização de pesquisa mais consistente com a rotina do engenheiro que, por vezes, precisa praticar seu lado de pesquisador.

Palavras-chave: Metodologias de pesquisa, Método Científico, Pesquisa em Engenharia. 


\section{INTRODUÇÃO}

Os problemas a serem resolvidos pela Engenharia são notadamente práticos. O engenheiro ao buscar a solução de uma situação problema, trabalhará, normalmente, aplicando ciência. Em outras palavras, no trabalho do engenheiro há ênfase no uso da ciência, não no desenvolvimento dela, assim como afirmado por Marconi e Lakatos (2003, p. 83) ao dizerem que todas as áreas da ciência usufruem de métodos científicos, porém nem todas que os utilizam são ciências.

O engenheiro não deve ser confundido com um cientista, na medida em que a Engenharia não se preocupa com a explicação do funcionamento de natureza, mas sim com a criação do artificial, ou seja, em sua rotina o engenheiro usa das descobertas da ciência e propõe a melhor solução possível para alterar um determinado estado de coisas.

Entretanto, a falta de métodos específicos que se apliquem diretamente à engenharia, dificulta a pesquisa daqueles que estudam a área, e os forçam a fazer adaptações de métodos correlatos para usar em seus estudos. Tendo isso, esta pesquisa tem como objetivo geral: descrever os métodos científicos que os engenheiros estão utilizando para realizar suas pesquisas. E no tocante aos objetivos específicos, buscou-se: 1. Analisar a ligação dos métodos de pesquisa existentes com a engenharia; 2. Inferir os métodos de pesquisa com maior aplicabilidade na engenharia; 3 . Investigar a dificuldade no entendimento do que seja a metodologia de pesquisa para a engenharia.

Reforçando a ideia, que segundo Leite (2009, p. 10), diz que metodologia científica vai além de um simples conteúdo que o acadêmico precisa decorar para realizar sua prova, mas é um ferramental imprescindível para conseguir seus objetivos na área acadêmica. O presente estudo se justifica de forma prática, por apresentar os Métodos Científicos mais usados e que possuem maior aplicabilidade na engenharia. Já no que diz respeito à justificativa teórica, entende-se que há uma lacuna na literatura sobre metodologia de pesquisa em engenharia, e faz-se necessário cada vez mais a fomentação de pesquisa nessa área, de forma a suprir as necessidades dos pesquisadores engenheiros.

Esse artigo foi estruturado da seguinte maneira: Introdução, por onde é apresentado o tema da pesquisa, bem como seus objetivos propostos; Referencial teórico, onde constam os conceitos utilizados ao longo do estudo, fundamentados na literatura; Metodologia, a qual apresenta o método utilizado para fazer a pesquisa; Resultados e discussões, por meio do qual os resultados obtidos são apresentados e discutidos; e por fim, as conclusões obtidas até o momento.

\section{DESENVOLVIMENTO}

Aqui, são descritos alguns conceitos utilizados no referencial teórico, baseados na literatura. Seguido da metodologia utilizada para a realização do estudo e, por conseguinte, uma sucinta demonstração dos resultados que foram encontrados.

\subsection{Referencial teórico}

Utilizar de métodos e meios para conseguir o que deseja é uma vantagem do engenheiro. Sua função, na maioria das vezes, é resolver problemas já instituídos ou em alguns casos trabalhar na prevenção para que eles não ocorram. Ser engenheiro é saber lidar e moldar o tangível para sua realidade, e cabe a ele estar preparado para as mais diversas situações, em qualquer lugar. Krick (1979, p. 24), diz que "a arte da engenharia compreende o engenho para imaginar as soluções e o discernimento, para compará-las", sendo assim, o papel do 
engenheiro vai muito além que somente resolver integrais e derivadas, mas em saber criar, desenvolver e aplicar soluções para os problemas do dia a dia.

Contudo, para todo projeto a ser feito, precisa-se de um método para sua realização. Nesse sentido, Krick (1979, p. 24) diz que o engenheiro tem várias maneiras de resolver seus problemas, seja usando seus conhecimentos adquiridos formalmente, seja de forma empírica, por meio de experiências anteriores. Fato esse, que é visto desde os primórdios da criação das primeiras ferramentas no período paleolítico, até o desenvolvimento de coisas maiores e por conseguinte no século XVI, onde essas ações ganharam o nome, em português, de Engenharia, oriunda do latim ingenium.

Tempos depois, vieram grandes acontecimentos históricos, como a Primeira Revolução Industrial, século XVIII; Primeira Guerra Mundial, em 1914; e posteriormente a Segunda Guerra Mundial, em 1939. Com isso, os conceitos de tecnologia passaram a ser fatores determinantes, para o desenvolvimento da sociedade. Nessa época - início do século XX -, o crescimento e desenvolvimento científico estavam em alta, e possibilitou que houvesse a junção entre conhecimento científico e técnicas, o que implicou em criações mais robustas, tais como novas fontes de energia através do motor a explosão (Veraszto et al., 2003).

Avançando para a metade do século XX, a engenharia ganhou novas especialidades, e consequentemente apareceram novas competências a serem desenvolvidas para a conquista do mercado de trabalho (Veraszto et al., 2003). Hoje ao falar sobre engenharia, engloba-se diversas áreas de estudos, desde as mais conhecidas, como civil, mecânica e química; até as menos conhecidas e recentes, como a engenharia matemática, criada pela UFRJ Universidade Federal do Rio de Janeiro.

Ao cursar engenharia, são duas as principais áreas que o graduando pode escolher para seguir: $1^{\circ}$ a acadêmica, e $2^{\circ}$ a industrial. Por muitos é atribuído que saber pesquisar é necessário somente para aqueles que decidem seguir a área acadêmica. Mas ao buscar a definição de pesquisa, percebe-se que há um equívoco nessa afirmação generalista. Gil (2002, p. 17), define pesquisa como sendo:

\footnotetext{
[...] o procedimento racional e sistemático que tem como objetivo proporcionar respostas aos problemas que são propostos. A pesquisa é requerida quando não se dispõe de informação suficiente para responder ao problema, ou então quando a informação disponível se encontra em tal estado de desordem que não possa ser adequadamente relacionada ao problema.
}

Sendo assim, uma pesquisa pode ser efetuada por todos os tipos de engenheiros independente de sua área de atuação, afinal, nem para todos os problemas há uma solução disponível. Nesse ponto, a aplicação de uma boa metodologia é imprescindível, para que se possa coletar os dados de forma eficaz e segura; bem como para conduzir o estudo de forma coerente aos objetivos da pesquisa.

Ao falar sobre metodologia, não se deve confundir com os métodos pedagógicos, a medida em que metodologia científica não tem a função de passar o conhecimento, mas sim em estudar analiticamente opções para desenvolver uma pesquisa (Ciribelli, 2003, p. 29 apud Asti-Vera, 1989; e Barros e Lehfeld, 1986). Ou seja, a metodologia cientifica diz respeito a utilização de métodos como: qualitativo, quantitativo, indutivo ou dedutivo, e não em ensinamentos. Pode-se então, definir metodologia, como sendo os procedimentos - isso inclui tanto as técnicas quando o ferramental - utilizados para conseguir resultados para um estudo (Ciribelli, 2003, p. 30).

Ao analisar historicamente o rumo que a engenharia vem tomando e suas dificuldades em relação a pesquisa, percebe-se que essas dificuldades vêm sido passadas de geração em geração, e nunca solucionadas por completo em sua fonte primária. Isso porque os problemas 
em engenharia são dos mais variados tipos, tornando sua delimitação e mensuração mais complexas. Isso tudo corrobora de forma negativa a desestimulação dos estudantes e pesquisadores da área.

Tem-se então, uma repulsa e dificuldade para elaborar textos acadêmicos instaurada ao perceber que não há um método de pesquisa específico para engenharia, e que os alunos e pesquisadores engenheiros acabam tendo de utilizar de métodos voltados para áreas diferentes, além de serem forçados a adaptarem esses métodos às suas necessidades. Situação essa descrita de acordo com Araújo e Santos (2001, p. 182) como um gap ao se tratar da formação de engenheiros, no que se refere a metodologias científicas.

Por mais que muitos dos métodos existentes não tenham cem porcento de aplicabilidade na engenharia, conhecê-los faz-se necessário à medida em que é preciso ter conhecimento teórico e base científica para condução de estudos mais profundos. Não é descartado nenhum método de pesquisa científica, porém é necessário procurar formas mais acuradas de se conseguir resultados para as pesquisas em engenharia.

Corroborando a ideia de Bazzo e Menestrina (2008, p. 6-7), os quais enfatizam a necessidade de se repensar sobre o ensino da engenharia, hoje essa necessidade ainda é mais evidente por se tratar da inserção de novos conceitos, como o da indústria 4.0. Conceito esse conhecido também como Quarta Revolução Industrial, o qual traz em seus fundamentos uma Revolução baseada em tecnologia. Isso reforça a importância de haver novas formas de se trabalhar com essas ideias as quais fazem parte do dia a dia do engenheiro.

Em suma, a utilização de métodos para um engenheiro é essencial, pois é a partir deles que são feitas as pesquisas para o desenvolvimento de novas coisas. Entretanto, cabe a ele fazer a avaliação correta do problema, utilizando de seu conhecimento científico, pois segundo Berto e Nakano (1999), tem-se um aumento da exigência por trabalhos fundamentados em ciência, dessa forma, é necessário um bom respaldo metodológico para que se tenha uma pesquisa de qualidade e com veracidade em suas informações.

\subsection{Metodologia}

Para realizar esse estudo, caracterizado como exploratório pois se trata de "uma primeira aproximação em relação ao tema e visa criar maior familiaridade em relação a um fato ou fenômeno" (SANTOS, 1999, p. 26), selecionou-se periódicos representativos das Engenharias de Produção e da Computação - visto que os pesquisadores atuam nessas áreas - publicados desde 2000 até 2019, através de fontes de pesquisa acadêmica como Scimago, Scielo, Dialnet, Worldwidescience, Scienceresearch, e Capes.

Para a coleta dos materiais foram utilizadas as seguintes expressões de busca: "metodologia de pesquisa", "metodologia científica", "metodologia de pesquisa em engenharia", "pesquisa cientifica em engenharia", "métodos de pesquisa acadêmica", "métodos de pesquisa científicos", "métodos científicos engenharia de computação" e "métodos científicos engenharia de produção".

A seleção dos materiais foi realizada a partir da utilização de dois indicadores que medem a qualidade de periódicos científicos: seu Qualis e seu fator de impacto quanto a publicações. O longo prazo relativo à análise longitudinal (19 anos) tem como explicação a necessidade de verificar se houve mudança ao longo do tempo no que se refere ao entendimento dos engenheiros quanto à metodologia.

Retirou-se dos periódicos as metodologias dos artigos publicados neles, por meio da técnica de fichamento, e fez-se uma análise caracterizando o tipo de pesquisa e os procedimentos metodológicos descritos nesses artigos, através de leitura analítica, que tem a finalidade de ajustar os dados para conseguir resultados aos problemas da pesquisa (Gil, 2002). Assim dizendo, uma leitura mais rigorosa das metodologias dos trabalhos, para 
analisar o que dentre elas tem maior aplicabilidade na pesquisa, bem como eliminar os artigos que não se enquadravam aos objetivos propostos.

Por fim, realizou-se uma última análise de forma geral das informações, por meio do método indutivo, que segundo Marconi e Lakatos (2003) visa proporcionar uma conclusão válida aos dados estudados no decorrer da pesquisa, isto é, com as informações extraídas na leitura analítica, fez-se uma avaliação panorâmica para quantificar o que era predominante na parte de metodologia dos artigos selecionados.

\subsection{Apresentação e discussão de resultados}

O que se pode observar com as informações coletadas, é que há duas vertentes nas descrições metodológicas: 1. Os autores descrevem o modo para conduzir ou solucionar o problema proposto em sua pesquisa; 2. Os autores realizam a descrição clássica de procedimentos metodológicos de pesquisa científica, caracterizando objetivos de pesquisa e procedimentos de coleta, tratamento e análise de dados. Vale ressaltar que não se observou alteração significativa desses dados no que se refere ao estudo longitudinal.

Em 75\% dos artigos encontra-se no capítulo Metodologia, a descrição dos procedimentos científicos tradicionais de metodologia de pesquisa. Desses artigos, metade apresentam metodologia semelhante entre si, com objetivo descritivo, usando de questionários e/ou entrevistas, por ser mais rápido e não exigir grandes recursos financeiros, no caso dos questionários, e poder ser aplicado em mais pessoas, no tocante as entrevistas (Gil, 2002, p. 115). Já para o tratamento e análise dos dados, 65\% dos artigos apresentam métodos estatísticos quantitativos, onde, de acordo com Wainer ([2007?], p. 6) "as variáveis observadas são poucas, objetivas e medidas em escalas numéricas", ou seja, os resultados serão sempre mais acurados, objetivos, e com relação numérica.

Outro procedimento comum à metade dos artigos analisados é a revisão sistemática de literatura, com posterior tratamento de dados quantitativo. A revisão sistemática do conhecimento surgiu na última década como alternativa a simples revisão de literatura e tem sido preferida pelos pesquisadores quando há necessidade da análise de uma questão específica, por meio da síntese dos resultados de vários estudos.

Por outro lado, em 25\% dos trabalhos analisados é apresentado no capítulo Metodologia, o método proposto de resolução ao problema, ao invés de se relatar como a pesquisa foi realizada. Erro este, que se dá pela sutil diferença de propósitos entre Resultados e discussões ${ }^{1}$, e Metodologia. A parte de Resultados e discussões, visa discorrer o método escolhido para a resolução do problema do estudo e como ele foi feito. Já em Metodologia, como outrora mencionado, tem por objetivo detalhar o tipo de pesquisa que foi escolhida, sua condução, e o ferramental escolhido para a coleta e tratamento dos dados da pesquisa.

\section{CONSIDERAÇÕES FINAIS}

Engenheiros e estudantes de engenharia contam com o desafio de criar mecanismos inéditos. No entanto, com o ensino a distância, a internet das coisas, e outras tecnologias conectando o mundo, parece haver uma sensação de que o conhecimento, que antes era armazenado na memória humana, é quase integralmente transferido para o mundo on-line. Vários estudantes ficam assim limitados por sua crença de que as respostas estarão no mundo digital. E quando se encontram exercendo plenamente a profissão tem como desafio utilizar dessas tecnologias, mas entendendo que, para obter o máximo de conhecimento, precisarão

\footnotetext{
${ }^{1}$ Também chamado de: Apresentação e discussão de resultados; ou Discussão de resultados.
} 
pesquisar e utilizar de métodos científicos que os resguardem de possíveis erros na solução dos problemas.

Entretanto, o que se percebe é que parece ser mais fácil para o engenheiro partir para fórmulas mais próximas à sua realidade, utilizando de métodos quantitativos e estatísticos, e objetivos descritivos em que se conta como o problema foi resolvido, do que proporcionar ao leitor de um artigo científico de Engenharia os passos dados para realizar a pesquisa, de maneira a fomentar a possibilidade de replicação do estudo e do incentivo para novas pesquisas.

Respondendo ao objetivo de pesquisa desse artigo, de descrever os métodos científicos que os engenheiros estão utilizando para realizar suas pesquisas, acredita-se que as metodologias encontradas na análise dos artigos científicos corroboram a suposição de que há dificuldade no entendimento do que seja a metodologia de pesquisa científica para a engenharia. No que diz respeito aos objetivos específicos, considera-se que foram alcançados, pois se conseguiu observar os métodos de pesquisa com maior aplicabilidade na engenharia, bem como a dificuldade no entendimento do que seja a metodologia de pesquisa para a área.

Conclui-se, assim, que se faz necessário estabelecer mais claramente a distinção entre metodologia científica de pesquisa e método de resolução de problemas já ao longo do curso de graduação, e estabelecer uma caracterização de pesquisa mais consistente com a rotina do engenheiro que, por vezes, precisa praticar seu lado de pesquisador. Observa-se também que há necessidade de levar esta pesquisa para uma próxima etapa, em que se proponham possíveis fórmulas metodológicas que façam sentido para a prática do engenheiro, sem deixar de lado o rigor tão necessário aos estudos científicos. Nesse sentido, vale afirmar que esse é um estudo em andamento e que novas pesquisas vêm sendo realizadas, na busca da proposição de uma Metodologia Científica que atenda às necessidades do engenheiro.

\section{REFERÊNCIAS}

ARAÚJO, Elenise Maria de; SANTOS, Fernando César Almada. Proposta de inclusão da disciplina "metodologia da pesquisa em engenharia de produção" na graduação da EESC - USP. In: Cobenge, 2001, p. 181-188.

BAZZO, Walter Antônio; MENESTRINA, Tatiana C. Ciência, tecnologia e sociedade e formação do engenheiro: análise da legislação vigente. R. B. E. C. T., Vol 1, $N^{\circ}$ 2, 2008, p. $1-18$.

BERTO, Rosa Maria Villares. S; NAKANO, Davi Noboru. A produção científica nos anais do encontro nacional de engenharia de produção: um levantamento de métodos e tipos de pesquisa. Prod., São Paulo, Vol. 9, N. 2, 1999, p. 65-75.

CIRIBELLI, Marilda Corrêa. Como elaborar uma dissertação de Mestrado através da pesquisa científica. Marilda Ciribelli Corrêa, Rio de Janeiro: 7 Letras, 2003.

GIL, Antonio Carlos. Métodos e técnicas de pesquisa social. 4.ed. São Paulo: Atlas, 2002.

KRICK, Edward V. Introdução à engenharia. Rio de Janeiro: Livros Técnicos e Científicos, 1979.

LAKATOS, Eva Maria; MARCONI, Marina de Andrade. Fundamentos de Metodologia Científica. 5.ed. São Paulo: Editora Atlas. 2003.

LEITE, F. H. C. Metodologia Científica. In: LEITE, F. H. C.; SAKAGUTI, S. T. Metodologia Científica/ Estatística II. Dourados - MS: UNIGRAN, 2009.

SANTOS, Antonio Raimundo dos. Metodologia científica: a construção do conhecimento. Rio de Janeiro: DP\&A editora, 1999. 
VERASZTO, Estéfano Viszconde et al. A Engenharia e os Engenheiros ao longo da História. In: XXXI CONGRESSO BRASILEIRO DE ENSINO DE ENGENHARIA. 2003. WAINER, Jacques et al. Métodos de pesquisa quantitativa e qualitativa para a Ciência da Computação. Instituto de Computação - UNICAMP, [2007?], p. 1-42.

\section{RESEARCH METHODOLOGY FOR ENGINEERING: AN ANALYSIS}

Abstract: This article, in its current phase, makes an analysis of the scientific methods most used by engineers and researchers in the field of Engineering, to do their research. For that, the most used methods were raised in the literature and the reason why they were chosen by most of the engineering researchers was analyzed. The question that drove the research was the following: "What scientific research methods are most used by engineering researchers?" From that, it was observed that most of the studies found in the literature, are made from questionnaires and / or interviews, quantitative statistical method, and by some, systematic literature review. It was also observed that there is some difficulty on the part of the engineering researchers and researchers in the area, in understanding the difference between the Methodology of scientific research and Results and discussions. Thus, it was concluded that there is a need to establish more clearly the distinction between scientific research methodology and problem solving method already during the undergraduate course, and to establish a research characterization more consistent with the routine of the engineer who, for sometimes you need to practice your researcher side.

Keywords: Research methodologies, Scientific Method, Engineering Research. 